\title{
Anti-foot-and-mouth disease virus effects of Chinese herbal kombucha in vivo
}

\author{
Naifang $\mathrm{Fu}^{1}$, Juncai $\mathrm{Wu}^{1}, \mathrm{Lv} \mathrm{Lv}^{2}$, Jijun $\mathrm{He}^{2}$, Shengjun Jiang ${ }^{1}$ \\ ${ }^{1}$ National Research Center for Cassava Processing and Development, \\ Tropical Crops Genetic Resources Institute, People's Republic of China. \\ ${ }^{2}$ National Foot and Mouth Diseases Reference Laboratory, Lanzhou Veterinary Research Institute, \\ People's Republic of China.
}

Submitted: August 17, 2014; Approved: February 16, 2015.

\begin{abstract}
The foot and mouth disease virus (FMDV) is sensitive to acids and can be inactivated by exposure to low $\mathrm{pH}$ conditions. Spraying animals at risk of infection with suspensions of acid-forming microorganisms has been identified as a potential strategy for preventing FMD. Kombucha is one of the most strongly acid-forming symbiotic probiotics and could thus be an effective agent with which to implement this strategy. Moreover, certain Chinese herbal extracts are known to have broad-spectrum antiviral effects. Chinese herbal kombucha can be prepared by fermenting Chinese herbal extracts with a kombucha culture. Previous studies demonstrated that Chinese herbal kombucha prepared in this way efficiently inhibits FMDV replication in vitro. To assess the inhibitory effects of Chinese herbal kombucha against FMDV in vitro, swine challenged by intramuscular injection with $1000 \mathrm{SID}_{50}$ of swine FMDV serotype $\mathrm{O}$ strain $\mathrm{O} / \mathrm{China} / 99$ after treatment with Chinese herbal kombucha were partially protected against infection, as demonstrated by a lack of clinical symptoms and qRT-PCR analysis. In a large scale field trial, spraying cattle in an FMD outbreak zone with kombucha protected against infection. Chinese herbal kombucha may be a useful probiotic agent for managing FMD outbreaks.
\end{abstract}

Key words: FMD, FMDV, chinese herbal kombucha.

\section{Introduction}

Foot-and-mouth disease virus (FMDV) infects cloven-hoofed animals and has had devastating consequences in many countries (Gibbens et al., 2001; Grubman et al., 2004; Knowles et al., 2001). Two strategies for its prevention and eradication have been widely adopted. Mass slaughter of animals at risk of infection has been used extensively in developed countries but economic factors have made vaccination more popular in developing countries where the prevalence of FMD is high (Li et al., 2010). Most countries in the European Union and North America have controlled the disease by slaughtering infected animals and those that may have been exposed, but it remains endemic in many African, Asian, and South American countries (Bayry et al., 2001). Although the slaughter policy has been highly successful in limiting the extent of outbreaks and eliminating the infection, it has important negative aspects.
Notably, because it is not used in conjunction with vaccination, the remaining herds have almost no residual immunity and so remain very vulnerable to the disease. New infections can therefore spread rapidly, particularly in densely populated areas (Bayry et al., 2001). The vulnerability of nominally FMD-free countries and regions is amply demonstrated by the recent outbreaks in Italy, Greece, the United Kingdom, Japan, and South Korea, among others (Bayry et al., 2001; Cottam et al., 2006; Ferguson et al., 2001; Muroga et al., 2012; Ryan et al., 2008; Sakamoto et al., 2002; Wee et al., 2004; Woolhouse et al., 2001; Yoon et al., 2012;). FMD has been successfully controlled by vaccination in many developing countries. However, existing FMDV vaccines have several drawbacks: they are expensive, have a narrow antigenic spectrum, provide only short term immunity, and are very fragile (Belsham et al., 2011; Kitching et al., 2007). 
Alternatives to the slaughter and vaccination strategies for FMD control have been investigated. These include RNAi-based treatments, in vivo interferon expression technology, the use of synthetic chemical inhibitors, and treatment with Chinese herbal extracts. RNA interference (RNAi) is an evolutionarily conserved mechanism of sequence-specific post-transcriptional gene silencing that is triggered by double-stranded RNA (dsRNA), including siRNA and miRNA, and has great potential for controlling viral infections (Du et al., 2011). Most studies on the use of RNAi to inhibit FMDV replication have focused exclusively on in vitro experiments using cellular models (Chen et al., 2004; Chen et al., 2006; Du et al., 2011; Kahana et al., 2004; Kim et al., 2008; Luo et al., 2011; Wang et al., 2008;), but there have been a few in vivo studies using large animal models such as swine (Chen et al., 2004; Chen et al., 2006; Luo et al., 2011; Wang et al., 2008). Interferons (IFNs) are the first line of the host's innate immune defenses against viral infection (Diaz-San Segundo et al., 2010). It has been demonstrated that type I, type II, and type III IFNs (IFN- $\alpha / \beta$ and IFN- $\gamma$, respectively) are active against many viruses (Basler et al., 2002; Diaz-San Segundo et al., 2010; Martin et al., 2012). A number of studies have investigated the ability of interferons to inhibit FMDV replication in vitro, and some of these have been extended into susceptible animal models such as guinea pigs, swine, and cattle. Vector-mediated interferon expression in vivo successfully inhibited FMDV replication and protected FMDV-challenged animals in some cases (Chinsanggaram et al., 2003; Diaz-San Segundo et al., 2010; Martin et al., 2012; Moraes et al., 2007). The broadspectrum efficacy of inoculation with combinational interferon-expressing vectors as a means of inhibiting FMDV replication in vivo and its ability to protect against FMDV infection in swine suggest that it may be more attractive than RNAi as a method of dealing with FMD outbreaks. However, the applicability of interferon-based FMD treatments may be restricted by their somewhat variable and inconsistent ability to protect cattle challenged with FMDV, concerns about the health of inoculated animals, and the appearance of resistant viral mutants. T-705 (favipiravir) and ribavirin are synthetic small molecules that inhibit FMDV reproduction by targeting a recently-identified pocket of the RNA-Dependent RNA Polymerase. Related compounds such as hypericin that are found in Chinese herbal broad-spectrum inhibitors of RNA viral infections have been studied as potential inhibitors of FMDV replication in vitro (Durk et al., 2010; Furuta et al., 2009; Sierra et al., 2007; Durk et al., 2010; Wang et al., 2006). Overall, despite the great potential of methods based on RNAi, interferons, and synthetic inhibitors of FMDV replication, none of these technologies have yet been proven to be effective at treating FMD in the field. As such, there is still a need for new and more efficient strategies for combating this disease.
FMDV is the type species of the Aphthovirus genus of the Picornaviridase family (Grubman et al., 2004). Unlike those of other picornaviruses, the FMDV capsid dissociates into separate pentameric subunits of around $12 \mathrm{~S}$ each at $\mathrm{pH}$ values below 6.5 (Brown et al., 1961). The virus is therefore sensitive to acidic conditions and can be deactivated by exposure to low pH environments (Bachrach et al., 1957). This instability is attributed to a cluster of His residues at the interface between the capsid's VP2 and VP3 subunits, which become protonated under acidic conditions, weakening the capsid due to electrostatic repulsion (Curry et al., 1995; Ellard et al., 1999). Acids such as peracetic acid and acetic acid effectively kill FMDV in vitro. However, in vivo acid treatment only inactivates FMDV rather than killing it, and has relatively short-lived effects. Acetic acid bacteria belonging to the genera Acetobacter and Gluconacetobacter are among the most effective producers of acetic acid in nature and are used to produce vinegar. If animals and their environments were sprayed with acetic acid bacteria as a protective measure in the event of an FMD outbreak, the bacteria would reproduce and generate acetic acid and other organic acids on an ongoing basis, potentially causing long-term inactivation of FMD in the sprayed area. Kombucha, a traditional Chinese drink made by fermenting sugared black tea with a symbiotic culture of acetobacter and yeast, is widely consumed throughout the world as a medicinal health-promoting beverage (Greenwalt et al., 2000). The $\mathrm{pH}$ of a typical kombucha batch is between 2.5 and 4.6 (Teoh et al., 2004), which is sufficiently acidic to inactivate FMDV in vitro.

Many broad-spectrum antiviral agents have been isolated from Chinese herbal plants. In an attempt to improve the utility of kombucha cultures in the treatment and suppression of FMDV in vivo, selected cultures were used to ferment aqueous extracts of four Chinese herbs known to produce such agents: licorice (Glycyrrhizae Radix), Luohanguo (Siraitia grosvenorii), Chrysanthemum (Dendranthema morifolium) and tea (Camellia sinensis). All of these plants are known to produce compounds with broad-spectrum antiviral activity and are widely considered to have beneficial effects on human health. Moreover, they are consumed extensively in China as edible medicinal plants. The primary active compound in licorice is glycyrrhizin, which reportedly shows broad-spectrum anti-viral activity against targets such as HIV, the Hepatitis A, B, and $\mathrm{C}$ viruses, the Herpesviridae family (VZV, HSV-1, EBS, and CMV), Flaviviruses, and viruses responsible for emerging diseases such as SARS (Hoever et al., 2005; Kumada et al., 2002;). Siraitia grosvenorii is a vine belonging to the genus Siraitia Merr, in the family Cucurbitaceae. It is endemic to southern China, where its fruit is known as Luohanguo and it has been cultivated for several centuries as a source of food and products used in beverages and traditional Chinese medicine. Notably, its fruit is reported to have beneficial effects in the treatment of dry coughs, sore 
throats, extreme thirst, and constipation. The primary active components of Luohanguo are the mogrosides, a group of terpene glycosides that are estimated to be about 300 times as sweet as sucrose (Kasai et al., 1989). The mogrosides produce a sweet taste in Chinese herbal Kombucha prepared using Luohango extracts and have been approved for use as dietary supplements in Japan, the United States, New Zealand and Australia (Qin et al., 2006). Chrysanthemum and green tea contain effective antiviral compounds such as chlorogenic acid and various polyphenolics, which can inhibit the reproduction of HIV, HBV, and the influenza virus, among others (Imanishi et al., 2002; Nakayama et al., 1993; Xu et al., 2008). The inhibitory effects of Chinese herbal kombucha against FMDV in vitro have been investigated in BHK21 cells and suckling mice. At a 1:4 dilution, Chinese herbal Kombucha was not toxic to BHK21 cells but inhibited the growth and reproduction of 1000 TCID $_{50}$ of FMDV in BHK21 cells. Similarly, a 1:2 dilution exhibited no toxicity towards 3-day-old suckling mice but effectively killed $1000 \mathrm{LD}_{50}$ of FMDV (Fu et al., 2014). This manuscript describes a series of experiments conducted to better characterize the in vivo activity of Chinese herbal kombucha mixtures against FMDV in swine.

\section{Materials and Methods}

\section{Cells and viruses}

Baby hamster kidney (BHK-21) cells were grown in Dulbecco's Modified Eagle's Medium (DMEM, GIBCO, Invitrogen Corporation, USA) supplemented with $10 \%$ heat-activated fetal bovine serum (FBS). The cultures were maintained at $37{ }^{\circ} \mathrm{C}$ in a $5 \% \mathrm{CO}_{2}$ humidified incubator $(\mathrm{Du}$ et al., 2011). FMDV isolates of strain O/China/99 (GenBank Accession number AF506822) were used for viral challenge in cells and suckling mice (Du et al., 2011; Zhang et al., 2004). The suckling mouse-passaged swine FMDV serotype $\mathrm{O}$ strain $\mathrm{O} / \mathrm{China} / 99$, an original and stronger challenge strain, was used in swine experiments and was obtained from the Experimental Animal Center of Lanzhou Veterinary Research Institute, Chinese Academy of Agricultural Sciences.

\section{Preparation and test of the Chinese herbal Kombucha}

The kombucha cultures employed in this work were purchased from the Beiking Food Research Institute and consisted mainly of Saccharomyces pastonanus and Acetobacter xylinum (Wu et al., 2004). The kombucha mother culture was grown in a nutrient broth containing $50 \mathrm{~g} / \mathrm{L}$ of sugar, $1 \mathrm{~g} / \mathrm{L}$ of $\left(\mathrm{NH}_{4}\right)_{2} \mathrm{SO}_{4}, 1 \mathrm{~g} / \mathrm{L}$ of $\mathrm{KH}_{2} \mathrm{PO}_{4}$, and $5 \mathrm{~g} / \mathrm{L}$ of tea. The resulting culture was stored at $4{ }^{\circ} \mathrm{C}$ until required for the preparation of the Chinese herbal kombucha used in the experiments (Murugesan et al., 2009). The experimental kombucha was prepared by mixing $50 \mathrm{~g}$ of sugar, $1 \mathrm{~g}$ of $\left(\mathrm{NH}_{4}\right)_{2} \mathrm{SO}_{4}$ and 1 gram $\mathrm{KH}_{2} \mathrm{PO}_{4}, 5 \mathrm{~g}$ of licorice
(Glycyrrhizae Radix), $2 \mathrm{~g}$ of Grosvenor Momordica (Momordica Grosvenori), $2 \mathrm{~g}$ of Chrysanthemum (Dendranthema morifolium) and $2 \mathrm{~g}$ of green tea (Camellia sinensis) in $1 \mathrm{~L}$ of distilled water. The resulting solution was boiled for $15 \mathrm{~min}$ in a sterile conical flask and then left to cool at room temperature for one hour. The cooled solution was filtered using a sterile nylon mesh, and the filtrate was inoculated with the Kombucha culture for fermentation. Fermentation was performed under aseptic conditions and was achieved by incubating the filtered solution with the Chinese herbal kombucha culture at $28 \pm 1{ }^{\circ} \mathrm{C}$ for 7 days. The Chinese herbal kombucha prepared in this way was stored at $4-8{ }^{\circ} \mathrm{C}$ until required for use in experiments. The heavy metal content of the prepared kombucha was determined using an ICPS7510 plasma emission spectrometer (Shimadzu, Japan). The number of Colony Forming Units (CFU) of acetic acid bacteria, yeasts, Escherichia coli, and Salmonella spp. per unit volume of the fermented kombucha was determined by the National Standard of Microbiological Examination of Food Hygiene of China (GB4789.2-94, China). The $\mathrm{pH}$ of the kombucha was determined using an FE20 pH meter (Shanghai Mettler Toledo, China).

\section{Efficacy of Chinese herbal kombucha against an FMDV challenge in vivo}

Approximately two-month-old healthy landrace swine (weighing 40-60 kg each) were acquired from a small swine farm in Zhuanglang County, Gansu province, China. All of the animals were free of FMDV infection, had not been vaccinated against the virus, and did not produce antibodies against FMDV. Twenty swine were divided into four groups of five animals each: a positive control group and high, intermediate, and low (H, I, and L) kombucha treatment groups. The animals in each group were cohoused in separate rooms. Three days prior to the viral challenge, the body temperatures of the animals in each group were determined. The positive control group was treated with a sucrose solution over a 13 day period that began three days before the viral challenge. The solution was prepared by mixing 3.01 of water with $150 \mathrm{~g}$ of sucrose and $3 \mathrm{~g}$ of $\mathrm{NaCl}$. Each animal was treated with 3.01 of this solution daily, with 2.01 being administered orally (i.e. provided as drinking water, with no other food offered) and the remaining 1.01 being administered by spraying the animal's mouth and nose using a 2.51 plastic sprayer. Similarly, animals in the H, M, and L groups were treated with 3.01 of a Chinese herbal kombucha solution daily for 13 days, starting three days before the viral challenge. The kombucha solutions were prepared by dissolving $150 \mathrm{~g}$ of sucrose and $3 \mathrm{~g}$ of NaCl in 3.01 of a mixture of water and the Chinese herbal kombucha stock solution. In the high strength treatment, the Chinese herbal kombucha stock solution was mixed with water on a 1:1 basis; for the intermediate strength treatment, the stock solution was mixed with water 
on a 1:3 basis; and for the low strength treatment, the two were mixed on a 1:11 basis. In each case, 2.01 of the resulting mixture was supplied as food (and thus administered orally), while the remaining 1.01 was administered by spraying the animal's mouth and nose. After three days of treatment with the appropriate sucrose or Chinese herbal kombucha solution, animals in all groups were inoculated with $1000 \mathrm{SID}_{50}$ of swine FMDV serotype $\mathrm{O}$ strain $\mathrm{O} / \mathrm{China} / 99$ in $3 \mathrm{~mL}$ of PBS by intramuscular injection in the ear-root-neck area. After this challenge, the animals were examined daily for clinical signs of FMD, including the formation of vesicles on the mouth and feet. The observations were terminated ten days after the initial challenge, at which point the animals were killed humanely. Blood and serum samples were collected on days $0,1,3,5$, and 7 after the challenge.

\section{Quantification of FMDV RNA in serum}

In order to detect FMDV replication in swine, total RNA was extracted from serum samples and analyzed by real-time quantitative reverse transcription-PCR (Q-RTPCR). FMDV RNA was isolated from serum samples using the RNeasy mini kit (Qiagen) according to the manufacturer's protocol. The RT-PCR experiments were performed using the ABI Prism 7700 sequence detector system (Applied Biosystems, Foster City, CA). All results are reported relative to a FMDV-positive serum sample provided by our diagnostic department. The following primers and probe were used: FMDV forward primer (5'CACTGGTGACAGGCTAAGG-3'), FMDV reverse primer (5'-CCCTTCTCAGATTCCGAGT-3'), and FMDV probe (5'-TGCCCTTCAGGTACCCCGAGGTAACA-3') (Alves et al., 2009). A foot-and-mouth disease virus realtime quantitative RT-PCR detection kit was provided by the Lanzhou Veterinary Research Institute of the Chinese Academy of Agricultural Sciences. To confirm specific amplification, a melting curve analysis of the RT-PCR products was performed according to the manufacturer's protocol. Serum samples were collected 3 days before the viral challenge, and on days 3 and 10 after the viral challenge.

\section{Quantification of neutralizing antibodies}

All swine serum samples were monitored by liquid-phase blocking sandwich ELISA performed according to the protocol supplied by the manufacturer of the O-type foot-and-mouth disease antibody liquid phase blocking ELISA kit used - the Lanzhou Veterinary Research Institute (LVRI), CAAS National Foot-and-Mouth Disease Reference Laboratory of P.R. China (Alves et al., 2009; Hamblin et al., 1986). Briefly, serum samples were inactivated by heating for $30 \mathrm{~min}$ at $56^{\circ} \mathrm{C}$ and then clarified by centrifugation. ELISA plates were coated with the optimal dilution of rabbit antisera against FMDV type Asia-I in carbonate/bicarbonate buffer $(\mathrm{pH} 9.6,1: 1000)$. The plate was then covered with sealplate film and allowed to stand overnight at room temperature. After incubation, the plates were drained and washed with PBST by filling and emptying all of their wells five times. Duplicate dilution series in PBST were then prepared from each serum sample in U-bottomed 96-well plates, with an initial dilution factor of 1 in 4 and a sample volume of $50 \mu \mathrm{L}$ in each well. $50 \mu \mathrm{L}$ of a viral solution of a fixed concentration, diluted in PBST (1:5), was then added to each well and the plate was shaken. The addition of the antigen thus increased the initial dilution factor of the serum samples to 1 in 8 . As controls, each plate also featured four wells containing only the virus diluted in PBST and positive serum, and four containing the virus solution plus negative serum. $50 \mu \mathrm{L}$ of each serum/virus mixture was then transferred from the carrier plate to an ELISA plate, which was then covered. After washing, $50 \mu \mathrm{L}$ of a guinea-pig antiserum solution against FMDV type Asia-I, at a dilution factor of 1:1000, was added to each well. The plates were then washed and $50 \mu \mathrm{L}$ of rabbit anti-guinea-pig immunoglobulin conjugated to horseradish peroxidase was added to each well, diluted 1:1000 in PBST. The plates were washed and $50 \mu \mathrm{L}$ of an o-phenylenediamine solution containing $\mathrm{H}_{2} \mathrm{O}_{2}(3 \% \mathrm{~W} / \mathrm{V})$ was added to each well. The ensuing reaction was stopped after $15 \mathrm{~min}$ by adding $1.25 \mathrm{M} \mathrm{H}_{2} \mathrm{SO}_{4}$. The plates were then read spectrophotometrically at $492 \mathrm{~nm}$.

\section{Field trial of Chinese herbal kombucha treatment as a method for controlling a small-scale FMD outbreak}

A small outbreak of foot and mouth disease occurred in 2010, affecting cattle herds owned by 21 farmers; in total, there were 582 cows that did not exhibit FMD symptoms within the outbreak area. This was used as an opportunity to test the utility of Chinese herbal kombucha treatment in controlling an FMD outbreak in the field. Based on the results obtained in vitro and in animal experiments, a 1:4 dilution of the kombucha stock solution was used in these studies. The 582 cows that did not exhibit FMD symptoms at the start of the trial were divided into two groups: an experimental group consisting of 314 cattle belonging to 10 farmers and a control group consisting of 268 cattle belonging to 11 farmers. The mouths and noses of the experimental group were sprayed with the diluted kombucha mix on a daily basis for six days, at a rate of $200 \mathrm{~mL}$ per day per animal. The control group was sprayed with the same volume of a $0.5 \%$ peracetic acid solution. After two weeks, the number of animals in both groups showing signs of FMD was determined.

\section{Statistical analysis}

Results are reported as means $\pm \mathrm{SD}$. The significance of differences between results for different groups were evaluated using one-way ANOVA with a p-value threshold of 0.05 . 


\section{Results}

\section{Preparation and tests of the Chinese herbal kombucha}

The Chinese herbal kombucha was prepared by fermenting Chinese herbal extracts with a kombucha culture that was rich in acetic acid bacteria and yeasts. Details of its composition are provided in Table 1. Importantly, the $E$. coli and heavy metal (e.g. mercury, lead, and arsenic) contents of the kombucha were within the limits specified in China's national standards for agricultural microbial agents (GB 20287-2006 GB). Moreover, the animal pathogen Salmonella spp. was not detected in any of the kombucha batches. The herbal kombucha had a very low $\mathrm{pH}$ of 2.75 , suggesting that the symbiotic micro organisms in the kombucha culture retained their strong acid-forming activity. However, the levels of acid bacteria and yeast in the kombucha are relatively low compared to those of other probiotic species, whose growth would be inhibited by the Chinese herbal extracts it contains. Consequently, it was not possible to fully evaluate the kombucha stock solution against the Chinese national standards for agricultural microbial agents (GB 20287-2006).

\section{Treatment with the Chinese herbal kombucha confers significant resistance against FMDV in swine}

Before the viral challenge, swine in the experimental groups treated with Chinese herbal kombucha and in the control group were apparently healthy and exhibited no obvious clinical symptoms. The kombucha dosages applied to the experimental groups were chosen on the basis of preliminary experiments in sucking mice. After three days of pre-treatment with either Chinese herbal kombucha or a sucrose solution as appropriate, the swine were challenged with $1000 \operatorname{SID}_{50}$ of the type O strain O/China/99 of the FMD virus. All of the control group animals (which were treated with a sucrose solution) exhibited the major clinical signs of FMD, including vesicle formation and diarrhea, within 3-4 days of being challenged, and one animal (no.1765) died from the infection (Table 2). Swine treated with the highest tested dose of Chinese herbal kombucha were not protected at all, and all animals in this group exhibited clinical symptoms within 2-3 days of being challenged. Only one of the animals treated with the intermediate dose of Chinese herbal kombucha was completely protected. However, three of five animals pretreated with the lowest dose of Chinese herbal kombucha developed none of the typical clinical symptoms such as vesicles. The lowest dose was thus the most effective of the tested treatments for FMDV in swine.

To assess the level of viral inhibition, blood samples from swine in the experimental and control groups were collected and analyzed by qRT-PCR to determine the number of FMDV genome copies they contained. None of the samples collected before the viral challenge contained any copies of the FMDV genome (Table 3). After the viral challenge, samples from the intermediate dose group contained the highest levels of FMDV. Samples from the high dose group contained higher levels of FMDV than did those from the control group, and the lowest viral loads were encountered in the low dose group. These results suggest that Chinese herbal kombucha has a dual effect on FMDV replication, stimulating its reproduction at high doses but inhibiting it at lower doses. At low doses, Chinese herbal kombucha efficiently inhibits FMDV replication in swine: samples from two animals in the low dose group contained no detectable viral genome copies. Treatment with Chinese herbal kombucha thus completely inhibited FMDV replication in the three asymptomatic animals from the low dose group and the single asymptomatic animal from the intermediate dose group. These results are consistent with those presented in Table 3. As shown in Table 4, one-way ANOVA indicated that the numbers of RNA virus copies in blood samples collected from the low dosage group on the first day differed significantly from those for the positive control (C) group, further demonstrating that treatment

Table 1 - Analytical data for the Chinese herbal kombucha stock solution

\begin{tabular}{|c|c|c|}
\hline Test sample & Test result & $\begin{array}{l}\text { Tolerable values according to the Chinese standards for agricultural microbial agents } \\
\qquad \text { (GB 20287-2006) }\end{array}$ \\
\hline $\mathrm{pH}$ value & 2.75 & \\
\hline Salmonella & Not detected & Not detected; see the animal feed requirements specified in Chinese Standard GB 13078-2001 \\
\hline E. coli & $<30 \uparrow / 100 \mathrm{~mL}$ & $<100 \uparrow / \mathrm{mL}$ \\
\hline Yeast & $4.9 \times 10^{6} / \mathrm{mL}$ & $\begin{array}{l}\geq 2.0 \times 10^{8} / \mathrm{mL} \text {. It is required that the effective number of viable cells for each active microbial } \\
\text { species be greater than } 1.0 \times 10^{6} \mathrm{~mL}^{-1} .\end{array}$ \\
\hline Acetic acid bacteria & $4.7 \times 10^{5} \mathrm{cfu} / \mathrm{mL}$ & See Chinese Standard GB 20287-2006 on agricultural microbial agents \\
\hline Mercury & Not detected & $\leq 0.1 \mathrm{mg} / \mathrm{kg}$ \\
\hline Lead & Not detected & $\leq 5.0 \mathrm{mg} / \mathrm{kg}$ \\
\hline Arsenic & Not detected & $\leq 2.0 \mathrm{mg} / \mathrm{kg}$ \\
\hline Other heavy metals & Not detected & $\leq 0.5 \mathrm{mg} / \mathrm{kg}$ \\
\hline
\end{tabular}


Table 2 - Clinical outcomes in swine treated with Chinese herbal kombucha and challenged with FMDV

\begin{tabular}{|c|c|c|c|c|c|c|c|c|c|c|c|c|}
\hline \multirow[t]{2}{*}{ Group } & \multirow[t]{2}{*}{ Animal number } & \multicolumn{10}{|c|}{ Clinical outcome per day } & \multirow{2}{*}{$\begin{array}{l}\text { No. animals protected/no. challenged at the } \\
\text { end of the experiment }\end{array}$} \\
\hline & & $1 \mathrm{~d}$ & $2 \mathrm{~d}$ & $3 d$ & $4 d$ & $5 \mathrm{~d}$ & $6 \mathrm{~d}$ & $7 \mathrm{~d}$ & $8 \mathrm{~d}$ & $9 \mathrm{~d}$ & $10 \mathrm{~d}$ & \\
\hline \multirow[t]{5}{*}{$\mathrm{H}$} & 2439 & - & + & + & + & + & + & + & + & + & + & $0 / 5$ \\
\hline & 2445 & - & - & + & + & + & + & + & + & + & + & \\
\hline & 2446 & - & - & + & + & + & + & + & + & + & + & \\
\hline & 2449 & - & + & + & + & + & + & + & + & + & + & \\
\hline & 2450 & - & + & + & + & + & + & + & + & + & + & \\
\hline \multirow[t]{5}{*}{ M } & 2044 & - & - & - & + & + & + & + & + & + & + & $1 / 5$ \\
\hline & 2045 & - & + & + & + & + & + & + & + & + & + & \\
\hline & 2046 & - & + & + & + & + & + & + & + & + & + & \\
\hline & 2047 & - & - & - & - & - & - & - & - & - & - & \\
\hline & 2048 & - & - & - & - & - & + & + & + & + & + & \\
\hline \multirow[t]{5}{*}{$\mathrm{L}$} & 2024 & - & - & - & - & - & - & - & + & + & + & $3 / 5$ \\
\hline & 2027 & - & - & - & - & - & - & - & - & - & - & \\
\hline & 2030 & - & - & - & - & - & - & - & - & - & - & \\
\hline & 2034 & - & - & + & + & + & + & + & + & + & + & \\
\hline & 2036 & - & - & - & - & - & - & - & - & - & - & \\
\hline \multirow[t]{5}{*}{ C } & 1764 & - & - & - & + & + & $\chi$ & & & & & $0 / 5$ \\
\hline & 1765 & - & - & + & + & + & + & + & + & + & + & \\
\hline & 1766 & - & - & - & - & + & + & + & + & + & + & \\
\hline & 1767 & - & - & - & + & + & + & + & + & + & + & \\
\hline & 1770 & - & - & - & + & + & + & + & + & + & + & \\
\hline
\end{tabular}

a-, Animal did not exhibit symptoms of FMD on the indicated day.

$\mathrm{B}_{+}$, Animal exhibited symptoms of FMD on the indicated day.

${ }^{\mathrm{C}} \chi$, Animal died on the indicated day.

with Chinese herbal kombucha at appropriate and practical dosages can effectively prevent the replication of the footand-mouth disease virus in vivo. However, there were no significant differences between the treatment groups on days 4-7. Thus, while the herbal kombucha treatment efficiently inhibited FMDV replication during the primary stage, it was less effective during the advanced stages of infection.

Serum samples were collected on the day of the viral challenge and also 3 and 10 days afterwards to test for the presence of neutralizing antibodies. Samples contained FMDV-specific antibodies at 1 or 3 days post-challenge (Table 5). Animals in the control groups treated with the sucrose solution developed clinical signs at 3-4 days postchallenge and exhibited a strong neutralizing antibody response at 10 post-challenge, suggesting exposure to very large amounts of FMDV. Because the high and intermediate does of Chinese herbal kombucha stimulated FMDV reproduction in vivo, very strong neutralizing antibody responses were detected in animals treated with these doses. Surprisingly, however, strong responses were also observed in samples taken from animals treated with the low dose of Chinese herbal kombucha, which did not exhibit FMD symptoms and whose serum samples contained few copes of the FMDV genome. Statistical analysis (ANOVA) indicated that there were no significant differences between the antibody responses of any of the tested groups. It therefore seems that while treatment with low doses of Chinese herbal kombucha prevented the replication of the FMD virus, it did not prevent the production of neutralizing antibodies.

\section{Clinical assessment of FMD control by spraying with Chinese herbal kombucha}

After two weeks, none of the animals in the experimental group exhibited symptoms of FMDV whereas 155 control group cows belonging to 4 different farmers did. Animals in the experimental group were thus completely protected by spraying with Chinese herbal kombucha. These field results clearly demonstrate the potential of Chinese herbal kombucha as a probiotic treatment for the prevention and containment of FMD outbreaks.

\section{Discussion}

Chinese herbal kombucha is prepared from two components: the kombucha culture and Chinese herbal extracts. The Chinese herbal kombucha used in this work had a $\mathrm{pH}$ 
Table 3 - Effects of Chinese herbal kombucha on the yield of FMDV in swine

\begin{tabular}{|c|c|c|c|c|c|c|}
\hline \multirow[t]{2}{*}{ Group } & \multirow[t]{2}{*}{ Animal NO } & \multicolumn{5}{|c|}{ FMDV RNA copies (copy/mL) } \\
\hline & & od & $1 d$ & $3 \mathrm{~d}$ & $5 \mathrm{~d}$ & $7 \mathrm{~d}$ \\
\hline \multirow[t]{5}{*}{$\mathrm{H}$} & 2439 & 0 & 1810 & 213400 & 32600 & 1450 \\
\hline & 2445 & 0 & 9060 & 0 & 0 & 0 \\
\hline & 2446 & 0 & 9756000 & 809020 & 1950 & 0 \\
\hline & 2449 & 0 & 149638160 & 106440 & 2370 & 0 \\
\hline & 2450 & 0 & 31360 & 2036410 & 3970 & 1820 \\
\hline \multirow[t]{5}{*}{ M } & 2044 & 0 & 2740 & 13415640 & 8880 & 0 \\
\hline & 2045 & 0 & 2658390 & 45539170 & 34570 & 8380 \\
\hline & 2046 & 0 & 7710268 & 5152608 & 2863 & 1793 \\
\hline & 2047 & 0 & 0 & 0 & 0 & 0 \\
\hline & 2048 & 0 & 0 & 0 & 59448230 & 18280 \\
\hline \multirow[t]{5}{*}{$\mathrm{L}$} & 2024 & 0 & 0 & 0 & 0 & 24717150 \\
\hline & 2027 & 0 & 0 & 1400 & 0 & 0 \\
\hline & 2030 & 0 & 0 & 0 & 0 & 0 \\
\hline & 2034 & 0 & 0 & 7140930 & 4640 & 0 \\
\hline & 2036 & 0 & 0 & 3220 & 0 & 0 \\
\hline \multirow[t]{5}{*}{$\mathrm{C}$} & 1764 & 0 & 232220 & 7975360 & 0 & - \\
\hline & 1765 & 0 & 3926580 & 9692780 & 4730 & 4150 \\
\hline & 1766 & 0 & 0 & 276770 & 19559640 & 0 \\
\hline & 1767 & 0 & 31970 & 3080 & 0 & 0 \\
\hline & 1770 & 0 & 1343310 & 692150 & 41470 & 14370 \\
\hline
\end{tabular}

${ }^{\text {a }}$, Animal died as a result of FMDV infection.

Table 4 - Comparative analysis of the effects of Chinese herbal kombucha on the yield of FMDV (copy/mL).

\begin{tabular}{lccccc}
\hline Groupldate & $\mathrm{d} 0$ & $\mathrm{~d} 1$ & $\mathrm{~d} 4$ & $\mathrm{~d} 5$ & $\mathrm{~d} 7$ \\
$\mathrm{C}$ & 0 & $1106816 \pm 1669749$ & $3728028 \pm 4706938$ & $3921168 \pm 8742189$ & $3704 \pm 6227$ \\
$\mathrm{H}$ & 0 & $31887278 \pm 65959774$ & $633054 \pm 844772$ & $8178 \pm 13725$ & $654 \pm 905$ \\
$\mathrm{~L}$ & 0 & $0 \pm 0 *$ & $1429110 \pm 3193005$ & $928 \pm 2075$ & $4943430 \pm 11053846$ \\
M & 0 & $2074280 \pm 3354180$ & $12821484 \pm 19094453$ & $11898909 \pm 26580882$ & $5691 \pm 7837$ \\
\hline
\end{tabular}

*: Value differs significantly from that for the positive control (C) group $(\mathrm{p}<0.05)$.

Table 5 - Serological analysis of samples from swine treated with Chinese herbal kombucha and challenged with FMDV

\begin{tabular}{lccc}
\hline Group $\backslash$ Date & $\mathrm{d} 1$ & $\mathrm{~d} 3$ & $\mathrm{~d} 10$ \\
\hline C & $8 \pm 0$ & $9.2 \pm 1.64$ & $352 \pm 414.84$ \\
H & $8 \pm 0$ & $27.2 \pm 35.63$ & $555.2 \pm 228.64$ \\
L & $9.2 \pm 1.64$ & $9.2 \pm 1.64$ & $441 \pm 427.9$ \\
M & $8.6 \pm 1.34$ & $9.2 \pm 1.64$ & $522 \pm 287.44$ \\
\hline
\end{tabular}

of between 2.75 and 4.5 , meaning that it was sufficiently acidic to kill FMDV directly. It therefore seems likely that some of the protective effects of kombucha treatment as observed in vitro are simply due to its organic acid content $(\mathrm{Fu}$ et al., 2014). However, the organic acid content of the kombucha used in this work would not be sufficient to substantially reduce the blood $\mathrm{pH}$ of the animals used in the in vivo experiments and the field trial presented herein, and therefore cannot explain the observed protective effects of kombucha treatment.

Instead, it is likely that either the microorganisms present in the kombucha or active components of the Chinese herbal extracts it contains are responsible for the suppression of FMDV replication in treated animals. When administered by intramuscular injection in swine, low doses of Chinese herbal kombucha conferred significant resistance to FMDV infection. Intermediate doses of kombucha conferred limited resistance, while high doses conferred no 
resistance. Similar trends were observed for the yields of FMDV RNA in blood samples taken from swine treated with kombucha and then exposed to FMDV. These results may indicate that kombucha treatment promotes viral replication at high doses but suppresses it at low doses. It is therefore important to determine the optimal dose for use in field trials before attempting to use kombucha to suppress FMDV outbreaks.

At present, the mechanism by which Chinese herbal kombucha exerts its antiviral effects remain unclear, and further research in this area will be required. The successful use of Chinese herbal kombucha to suppress an FMD outbreak in the field may indicate that the Chinese herbal extracts it contains have some antiviral activity that prevents FMDV replication in vivo. Based on the difference between the results observed in the experiments with swine and those observed in the field trial using cattle, it appears that Chinese herbal kombucha is more effective at preventing FMDV infections in cattle. It is possible that there is only one mechanism by which kombucha inhibits FMDV replication in swine but that there are additional such mecha- nisms operating in cattle (Figure 1). Since Chinese herbal kombucha is capable of directly inactivating FMDV in vitro, it is likely that simply spraying the kombucha mixture on the cattle would provide a first line of defense by effectively disinfecting the animals and their environment. Second, in the field trial, the kombucha was administered directly onto the cows' respiratory and digestive tracts by spraying it into their mouths and noses. The acetic acid bacteria and yeast in the Chinese herbal kombucha may be able to survive in the animal's digestive and respiratory tracts, where they would reproduce and produce organic acids. Together with the Chinese herbal extracts in the kombucha, this production of acids may contribute to the inactivation of FMDV or the suppression of its replication, providing a second line of defense. Finally, if some quantity of the virus were to enter into the animal's circulatory system, its reproduction could be inhibited by any active components of the Chinese herbal kombucha circulating in the animal's blood, thereby providing a third line of defense. The results obtained in this work provide evidence for the existence of the first and third lines of defense. Moreover, the successful

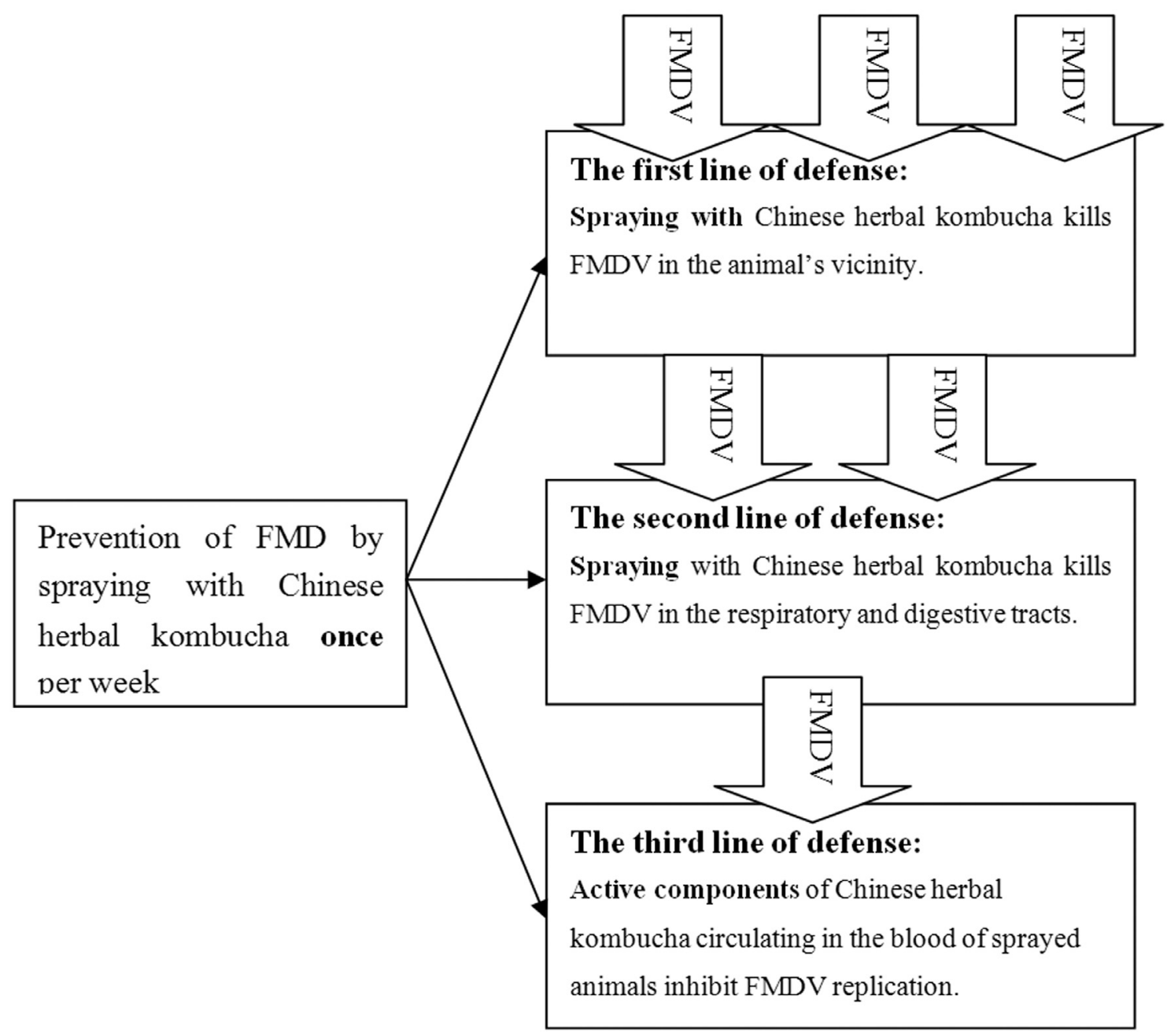

Figure 1 - Mechanisms by which Chinese herbal kombucha spraying prevents FMD. 
field trial provides some support for the existence of the second. The large scale field trial of treatment with Chinese herbal kombucha as a preventative treatment for FMD in cattle was conducted in 2011 (see the supplementary material) while the small scale experiments on the use of Chinese herbal kombucha were conducted between 2010 and 2012 on three small farms (see the supplementary material). All of these clinical investigations suggested that spraying with Chinese herbal kombucha may be an effective method for preventing and controlling FMD.

At present, there are five main strategies used to control FMD: slaughter, vaccination, RNAi technology, interferon-based approaches, and methods based on the use of synthetic inhibitors of FMDV replication (Diaz-San Segundo et al., 2010; Du et al., 2011; Durk et al., 2010; Basler et al., 2002; Furuta et al., 2009; Li et al., 2010; Martin et al., 2012; Sierra et al., 2007; Wang et al., 2006). Spraying with Chinese herbal kombucha has a number of advantages relative to all of these strategies (Figure 1). First, Chinese herbal komubucha spraying protects against FMDV infection in several ways as a result of the inhibitory effects of organic acids on viral replication and the potential antiviral effects of the Chinese herbal extracts used in its production. Second, Chinese herbal komubucha spraying kills or inhibits FMDV both within the animal and in its environment, and in vitro experiments have shown that some microorganisms in the kombucha culture (acetic bacteria and yeast) continue reproducing after application, producing acids and killing or inhibiting the reproduction of FMDV. Third, kombucha is likely to have broad spectrum antiviral activity against FMDV because its mechanisms of inhibition stem from its organic acid content and various active compounds produced by four different plants. As such, it is unlikely that any FMD mutant would be resistant to all of its effects. Fourth, herbal kombucha treatment can be used as a routine protective or preventative measure against FMD infection but is also suitable for use in emergencies. At present, only developed countries use large scale slaughter to deal with FMD infections. However, in the long term, many developing countries such as China would like to abandon the vaccination approach and instead use slaughter to establish FMD-free regions. Spraying with Chinese herbal kombucha represents a non-vaccination strategy that can prevent FMD and could thus be useful for suppressing outbreaks of the disease during the transition from vaccination to slaughter as the preferred strategy. In FMD-free countries that do not use vaccination, Chinese herbal kombucha spraying could be used to help control FMD outbreaks and minimize the need for slaughter, especially in countries with high animal feeding densities such as Japan and South Korea. By using kombucha spraying in conjunction with slaughter and vaccination, it may be possible to establish large FMD-free regions around the world, significantly increasing global food security.

\section{Acknowledgments}

This research was supported in part by earmarked funds from the China Agriculture Research System (CARS-32-hnjsj) and the basic scientific fund of the Tropical Crops Genetic Resources Institute at the Chinese Academy of Tropical Agriculture Science (009).

\section{References}

Alves MP, Guzylack-Piriou L, Juillard V et al. (2009) Innate immune defenses induced by $\mathrm{CpG}$ do not promote vaccineinduced protection against foot-and-mouth disease virus in pigs. Clin Vaccine Immunol 16:1151-1157.

Bachrach HL, Breese SS, Callis JJ et al. (1957) Inactivation of foot-and-mouth disease virus by $\mathrm{pH}$ and temperature changes and by formaldehyde. Proc Soc Exp Biol Med 95:147152.

Belsham GJ, Jamal SM, Tjørnehøj K et al. (2011) Rescue of foot-and-mouth disease viruses that are pathogenic for cattle from preserved viral RNA samples. PLoS One 6:e14621.

Basler CF, Garcia-Sastre A (2002) Viruses and the type I interferon antiviral system: induction and evasion. Int Rev Immunol 21:305-337.

Bayry J, Kaveri S (2001) Foot and mouth disease: a revised policy is required. J Clin Microbio 39:3808.

Brown F, Cartwright B (1961) Dissociation of foot-and-mouth disease virus into its nucleic acid and protein components. Nature 192:1163-1164.

Chen W, Liu M, Jiao Y et al. (2006) Adenovirus-mediated RNA interference against foot-and mouth disease virus infection both in vitro and in vivo. J Virol 80:3559-3566.

Chinsangaram J, Moraes MP, Koster M et al. (2003) The Chinese herbal viral disease control strategy: adenovirus expressing alpha interferon rapidly protects swine from foot-and-mouth disease. J Virol 77:1621-1625.

Cottam EM, Haydon DT, Paton DJ et al. (2006) Molecular epidemiology of foot-and-mouth disease virus outbreak in the United Kingdom. J Virol 80:11274-11282.

Curry S, Abrams CC, Fry E et al. (1995) Viral RNA modulates the acid sensitivity of foot-and-mouth disease virus capsids. J Virol 69:430-438.

Diaz-San Segundo F, Moraes MP, de los Santos T et al. (2010) Interferon-induced protection against foot-and-mouth disease virus infection correlates with enhanced tissue-specific innate immune cell infiltration and interferon-stimulated gene expression. J Virol 84:2063-2077.

Du J, Gao S, Luo J et al. (2011) Effective inhibition of foot-andmouth disease virus (FMDV) replication in vitro by vector-delivered microRNAs targeting the $3 \mathrm{D}$ gene. Virol $\mathrm{J}$ 8:292.

Durk RC, Singh K, Cornelison CA et al. (2010) Inhibitors of Foot and Mouth Disease Virus Targeting a novel Pocket of the RNA-Dependent RNA Polymerase. PLoS One 5:e15049.

Ellard FM, Drew J, Blakemore WE et al. (1999) Evidence for the role of His-142 of protein $1 \mathrm{C}$ in the acid-induced disassembly of foot-and-mouth disease virus capsids. J Gen Virol 80:1911-1918.

Ferguson NM, Donnelly CA, Anderson RM (2002) The footand-mouth epidemic in Great Britain: pattern of spread and impact of interventions. Science 292:1155-1160. 
Fu N, Wu J, Fu Q et al. (2014) Chinese herbal Kombucha preparation and its anti-viral activity of FMDV in vitro. J Anhui Agri Sci 42:5500-5502.

Furuta Y, Takahashi K, Shiraki K et al. (2009) T-705 (favipiravir) and related compounds: The novel broad-spectrum inhibitors of RNA viral infections. Antiviral Res 82:95-102.

Gibbens JC, Sharp CE, Wilesmith JW et al. (2001) Descriptive epidemiology of the 2001 foot-and-mouth disease epidemic in Great Britain: the first five months. Vet Rec 149:729-743.

Greenwalt CJ, Steinkraus KH, Ledford RA (2000) Kombucha, the fermented tea: microbiology, composition, and claimed health effects. J Food Prot 63:976-981.

Grubman MJ, Barry B (2004) Foot-and-mouth disease. Clin Microbiol Rev 17:465-493.

Hamblin C, Barnett IT, Crowther JR (1986) A new enzyme-linked immunosorbent Assay (ELISA) for the detection of antibodies against foot-and-mouth disease virus. J Immunol Methods 93:123-129.

Hoever G, Baltina L, Michaelis M et al. (2005) Antiviral activity of glycyrrhizic acid derivatives against SARS-coronavirus. J Med Chem 48:1256-1259.

Imanishi N, Tuji Y, Katada Y et al. (2002) Additional inhibitory effect of tea extract on the growth of influenza A and B viruses in MDCK cells. Microbiol Immunol 46:491-494.

Kahana R, Kuznetzova L, Rogel A et al. (2004) Inhibition of foot-and-mouth disease virus replication by small interfering RNA. J Gen Virol 85:3213-3217.

Kasai R, Nie RL, Nashi K et al. (1989) Sweet cucurbitane glycosides from fruits of Siraitia siamensis (chi-zi luo-han-guo) a Chinese folk medicine. Agric Biol Chem 53:3347-3349.

Kim SM, Lee KN, Park JY et al. (2008) Therapeutic application of RNA interference against foot-and-mouth disease virus in vitro and in vivo. Antiviral Res 80:178-184.

Kitching P, Hammond J, Jeggo M et al. (2007) Global FMD control ${ }^{\mathrm{a}}{ }^{\mathrm{i}}$ is it an option? Vaccine 25:5660-5664.

Knowles NJ, Samuel AR, Davies PR et al. (2001) Outbreak of foot-and-mouth disease virus serotype $\mathrm{O}$ in the UK caused by a pandemic strain. Vet Rec 148:258-259.

Kumada H (2002) Long-term treatment of chronic hepatitis C with glycyrrhizin stronger neo-minophagen C (SNMC) for preventing liver cirrhosis and hepatocellular carcinoma. Oncology 62:94-100.

Li D, Lu ZJ, Xie BX et al. (2010) Alternative way to test the efficacy of swine FMD vaccines: measurement of pigs median infected dose (PID50) and regulation of live virus challenge dose.Virol J 7:215.

Luo J, Du J, Gao S et al. (2008) Lentviral-mediated RNAi to inhibit target gene expression of the porcine integrin $\mid$ Áv subunit, the FMDV receptor, and against FMDV infection in PK-15 cells. Virol J 8:428.

Moraes MP, de Los Santos T, Koster M et al. (2007) Enhanced antiviral activity against foot-and-mouth disease virus by a combination of type I and II porcine interferons. J Virol 81:7124-7135

Muroga N, Hayama Y, Yamamoto T et al. (2012) The 2010 foot-and-mouth disease epidemic in Japan. J Vet Med Sci 74:399-404.

Murugesan GS, Sathishkumar M, Jayabalan R et al. (2009) Hepatoprotective and curative properties of Kombucha tea against carbon tetrachloride-induced toxicity. J Microbiol Biotechnol 19:397-402.
Nakayama M, Suzuki K, Toda M et al. (1993) Inhibition of the infectivity of influenza virus by tea polyphenols. Antiviral Res 21:289-299.

Perez-Martin E, Weiss M, Diaz-San Segundo F et al. (2012) Bovine type III interferon significantly delays and reduces the severity of foot-and-mouth disease in cattle. J Virol 86:4477-4487.

Qin X, Xiaojian S, Ronggan L et al. (2006) Subchronic 90-day oral (Gavage) toxicity study of a Luo Han Guo mogroside extract in dogs. Food Chem Toxicol 44:2106-2109.

Ryan E, Gloster J, Reid SM et al. (2008) Clinical and laboratory investigations of the outbreaks oif foot-and-mouth disease in southern England in 2007. Vet Rec 163:139-147.

Sakamoto K, Kanno T, Yamakawa M et al. (2002) Isolation of foot-and-mouth disease virus from Japanese black cattle in Miyazaki Prefecture, Japan 2000. J Vet Med Sci 64:91-94.

Sierra M, Airaksinen A, Gonzølez-L®pez C et al. (2007) Footand-mouth disease virus mutant with decreased sensitivity to ribavirin: implications for error catastrophe. J Virol 81:2012-2024.

Teoh AL, Heard G, Cox J (2004) Yeast ecology of Kombucha fermentation. Int J Food Microbiol 95:119-126.

Wang P, Ren Y, Guo Z et al. (2008) Inhibition of foot-and-mouth disease virus replication in vitro and in vivo by small interfering RNA. Virol J 5:86.

Wang S, Liang J, Yang L et al. (2006) Studies on the inhibitory effect of hypericin on foot-and-mouth virus in vitro. Chin J Veter Med 42:6-9.

Wee SH, Park JY, Joo YS et al. (2004) Control measures implemented during the 2002 foot-and-mouth disease outbreak in the Republic of Korea. Vet Rec 154:598-600.

Woolhouse M, Chase-Topping M, Haydon D et al. (2001) Footand-mouth disease under control in the UK. Nature 411:258-259.

Wu W, Gai B, Ji B (2004) Study on the Isolation and Identification of Microbes of Kombucha. Food Sci 25:55-58.

Xu J, Wang J, Deng F et al. (2008) Green tea extract and its major component epigallocatechin gallate inhibits hepatitis B virus in vitro. Antiviral Res 78:242-249.

Yoon H, Yoon SS, Wee SH et al. (2012) Clinical Manifestations of Foot-and-Mouth Disease During the 2010/2011 Epidemic in the Republic of Korea. Transbound Emerg Dis 59:517525.

Zhang XS, Liu ZX, Zhao QZ et al. (2004) Sequencing and analysis for the full-length genome RNA of foot-and-mouth disease virus China/99. Sci China Ser C Life Sci 47:74-81.

\section{Appendix}

\section{Clinical experiment on the prevention of FMD by spraying with Chinese herbal kombucha}

A small foot and mouth disease outbreak occurred in China in 2010. A field trial of the efficacy of kombucha treatment in preventing FMD infection was initiated at a point where there were 582 cows (belonging to 21 different farmers) within the affected region that did not exhibit FMD symptoms. The healthy cows were separated into a test and a control group. Based on the results of the previously-described in vitro and in vivo experiments, the test group (which consisted of 314 cows belonging to 10 farm- 
ers) was treated with a 1:4 dilution of Chinese herbal kombucha, administered by daily spraying on the nose and mouth over a six day period. The control group (268 cows belonging to 11 farmers) was instead treated with a $0.5 \%$ solution of peracetic acid. After two weeks, none of the animals in the experimental group exhibited symptoms of FMDV whereas 155 cows belonging to 4 farmers of those in the control group did. Animals in the experimental group were thus completely protected by spraying with Chinese herbal kombucha. These field results clearly demonstrate the potential of Chinese herbal kombucha as a probiotic treatment for the prevention and containment of FMD outbreaks.

Information concerning the field experiment on the use of Chinese herbal kombucha spraying to control a large FMD outbreak

In 2011, a large amount of Chinese herbal kombucha was distributed to farmers who collectively owned around 200000 swine and cattle in an area that had suffered an FMD outbreak in 2010. The 1:4 diluted Chinese herbal kombucha was sprayed on swine and cattle, and no further cases of FMD were reported in 2011. In 2012-2013, the local government abandoned the practice of distributing Chinese herbal kombucha to farmers in the region, and some FMD cases were reported. This suggests that Chinese herbal kombucha spraying could be useful in preventing FMD infections on a large scale.

Information concerning the field experiment on the use of Chinese herbal kombucha spraying to control FMD in small swine farms

Between 2010 and 2012, farmers at three small farms in different locations continuously sprayed their animals with a 1:4 dilution of Chinese herbal kombucha on a weekly basis to prevent FMD. No FMD cases in their swine were reported, despite the incidence of FMD cases at nearby farms. This result demonstrates the usefulness of Chinese herbal kombucha in preventing FMD outbreaks on smaller scales.

Associate Editor: João Pessoa Araújo Junior

All the content of the journal, except where otherwise noted, is licensed under a Creative Commons License CC BY-NC. 